\title{
Design and Fabrication of Remote-Controlled Nursing Vehicle
} Gopal Kaliyaperumal ${ }^{1}$, Choudhury Rajat Kumar Pattnaik², Akash Kumar Nath ${ }^{3}$, Aditya Dubey ${ }^{4}$, Tarun Kumar Sharma ${ }^{5}$

${ }^{1}$ Associate Professor, Department of Mechanical Engineering, New Horizon College of Engineering, Bangalore, Karnataka, India

2,3,4,5 UG Scholar, Department of Mechanical Engineering, New Horizon College of Engineering, Bangalore, Karnataka, India

Article Info

Volume 8, Issue 4

Page Number: 188-198

Publication Issue :

July-August-2021

\section{Article History}

Accepted : 10 July 2021

Published: 15 July2021

\section{ABSTRACT}

The need for remote controlled vehicle which has functions like a robot, to ensure proper monitoring of patients, delivering of needed kits and keeping the hospital environment sanitized, even in the absence of human being, is a serious demand in these times of Covid pandemic. We therefore intend to provide a solution by constructing a nursing vehicle which can be used in hospital surroundings. The project involves use of temperature sensor, water pump, microcontroller, Wi-Fi camera and embedded C language program. The project is locally controlled using ESP8266 based embedded system. The nursing vehicle's movements are controlled using a Wi-Fi controller and monitored with the help of camera which also has voice kit that can be used for interaction between patients and doctors. The MLX90614 IR temperature sensor is also fitted helping in measuring the temperature of patients. This also has a provision for sanitizing the patient or surroundings using the nozzle and pump kit which is an added advantage in this model. This project provides very stable and reliable system and significantly limits the drawbacks faced in hospital environment. The nursing vehicle will facilitate contactless temperature measurement, delivery of supplies to patients suffering from contagious diseases such as Covid and enables communication between a doctor and a patient adhering to social distancing norms. With this system we can tackle the problems of human resource shortage in hospitals and can be used as substitute for nurse.

Keywords: Automation, Healthcare, Nursing Vehicle, Engineering and Technology

\section{INTRODUCTION}

There has been much logical advancement in the field of manufacturing in the modern world. Advanced innovations that have improved human life have increased with increasing living standards. Engineers are engrossed in creative work. Year after year, researchers have created better ideas that have resulted in more mechanized processes. As part of an effort to construct a strategy set up in any area conceivable which would additionally streamline and optimize human life, we wound up with the hugely vital area of concern that is Healthcare.

Hospitals, Clinics, Small scale Check-up rooms, Pharmacy, and so on are examples of places where healthcare is possibly the most important factor transcendentally. However, due to the limited reach 
of computerization in these sectors, the idea of automated healthcare in such fields should be taken seriously. Along these lines, to broaden the benefits of automated healthcare in such fields should be given careful consideration. As a result, to extend the benefits of automated healthcare in large-scale areas to the previously mentioned division, an automation idea has been conceived to execute in small scale divisions.

\section{METHODS AND MATERIAL}

\section{A. Methodology}

The components that will be utilized in these projects are 3D designed and drafted using the Catia V5 application based on the measurements. This provides us an idea of how the model will look when we've assembled all the components using the dimensions we've chosen. The motion steering utilized in this project must convey items and perform many duties from one location to another, automobile wiper motors with configured clockwise and anti-clockwise rotation are used for simple operation in turning the vehicle. For patient temperature monitoring, an infrared sensor is utilized, which provides real-time temperature without the requirement for touch detection and is not impacted by oxidation or corrosion. For contact-free communication between doctor and patient, as well as sanitization, a Wi-Fi Camera with speaker and a $12 \mathrm{~V}$ Spray Pump are utilized. Steel is utilised for the chassis, which is securely welded according to measurements, and the vehicle is covered with thin plywood. Because they are user-friendly and easily configurable, ESP8266 controllers with Wi-Fi modules are utilised for local control. The Vehicle Chassis structure is first built, followed by the integration of the battery, motor, pump with nozzle, and camera. The ESP8266 is linked to the IR sensor that is used to sense, as well as relays that regulate the switching of the pump and motors. The BLYNK open-source Android application is used to remotely operate all vehicle operations. The prototype of the model is created by gathering materials, geometric 3D modelling of the project, and assembling the parts. The code required for measuring temperature using IR sensors must be uploaded into the ESP8266 node MCU, along with the software written using the Arduino IDE platform. Following the completion of the programming, the right connections between the hardware are established. The BLYNK open-source Android application is used to remotely operate all vehicle operations.

\section{B. Components Used}

\section{ESP8266}

The ESP8266 is a System on a Chip (SoC) made by Espressif, a Chinese firm. A Tensilica L106 32-bit micro controller unit (MCU) and a Wi-Fi transceiver make up the device. It features 11 GPIO pins* (General Purpose Input/Output pins) as well as an analogue input. It's a solution for the Internet of Things market that satisfies users' constant requests for economic power consumption, small design, and dependable performance. The ESP8266EX can function as a standalone application or as a slave to a host Microcontroller due to its complete and selfcontained Wi-Fi networking capabilities. When the application is hosted by the ESP8266EX, it starts up right away from the flash. The embedded high-speed cache aids in improving system performance and memory optimization. ESP8266EX can also be used as a Wi-Fi adaptor in any microcontroller architecture through Interfaces such as SPI/SDIO or UART. Antenna switches, RF baluns, power amplifiers, filters, low noise receive amplifiers, and power management modules are all included into the ESP8266EX. The small design reduces PCB size and eliminates the need for external circuitry. Aside from Wi-Fi capabilities, the ESP8266EX also includes an improved version of L106 Diamond series 32-bit processor with on-chip SRAM from Tensilica. It can be connected to other 
devices. Through the GPIOs, you can communicate with external sensors and other devices. Software Development kit (SDK) contains sample code for a few applications.

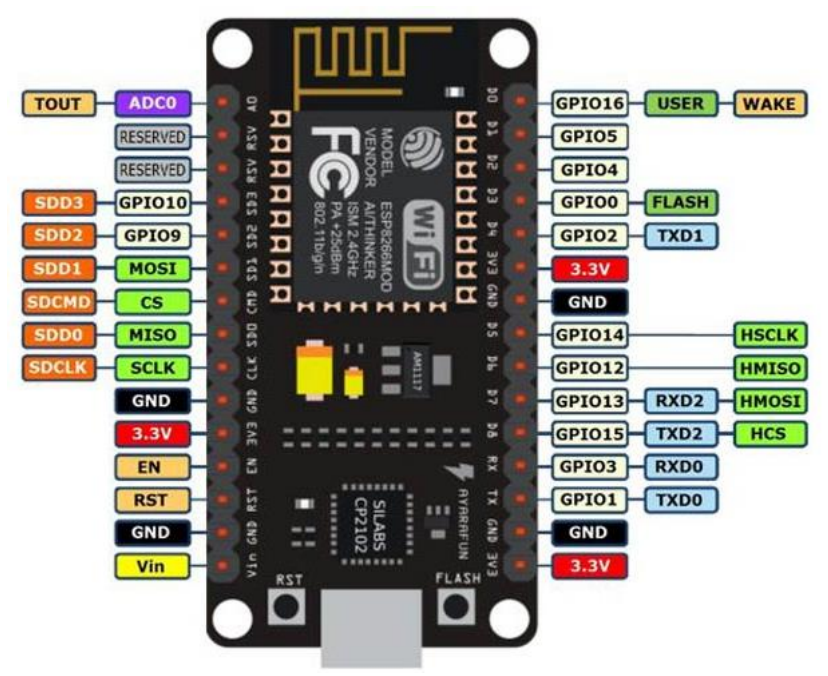

Figure 1: ESP8266 Node MCU

\section{Wheel Motor}

A wiper motor is made up of three major parts: the motor, the rotary mechanism for linear motion converter, and the switch for parking. The mechanism for converting the conversion of rotary motion to linear motion is simple, and its functionality can be seen by inspecting a motor assembly that has been disassembled. When you activate the wiper, the wiper switch sends a signal to the control module. The wiper relay is controlled by the control module. The relay supplies 12 volts to the wiper motor. The motor turns a small arm, which moves the wiper arms via links.

\section{A) NORMAL OPERATION:}

In this mode of operation, the dash switch is in the normal, or low speed, position., and switch of terminal 2 is internally linked to terminal 3 . The dotted red line depicts the flow of current through the motor. The parking switch has no effect in this mode because the dash switch's terminal 4 is not linked to any other end.

\section{B) HIGH SPEED OPERATION:}

In this mode the switch of the dash is in high speed, and the flow of current is shown. This is essentially the same setting as normal mode, with the exception that power is routed using a high-speed brush rather than a regular-speed brush. Terminal $2^{\text {nd }}$ switch of dash is internally linked to terminal $1^{\text {st. }}$.

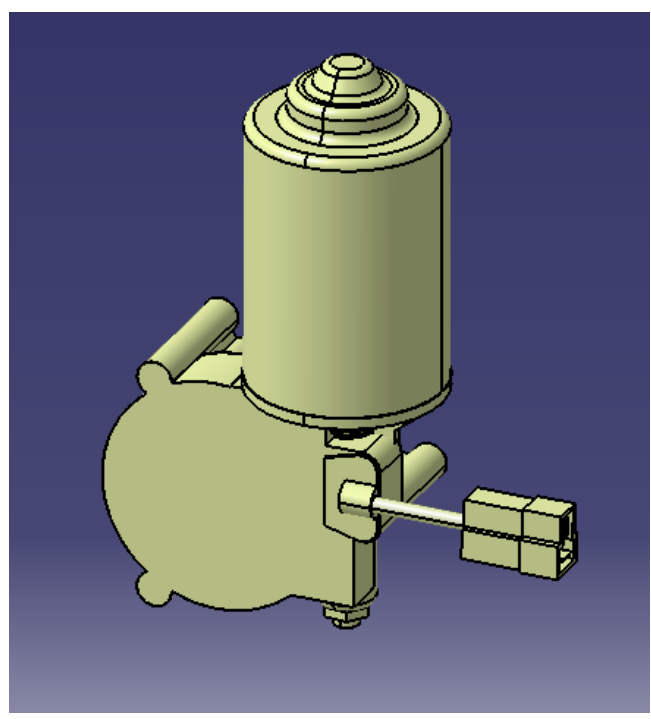

Figure 2: 12V Wheel Motor

\section{Relay}

A relay can be thought of as a switch. Switches are typically used to manually close or open a circuit. A relay, like a switch, connects or disconnects two circuits. Instead of manual operation, an electrical signal is applied to a relay, which connects or disconnects another circuit. The principle of electromagnetic induction underpins the operation of a relay. When a current is applied to an electromagnet, it creates a magnetic field around it. The image depicts the relay in action. To apply DC current to the load, a switch is used. In the relay, an electromagnet is formed by a copper coil and an iron core. When a DC current is applied to the coil, it begins to attract the contact as shown. This is known as relay energising. When the supply is removed, it returns to its original location. This is known as relay de-energizing. 


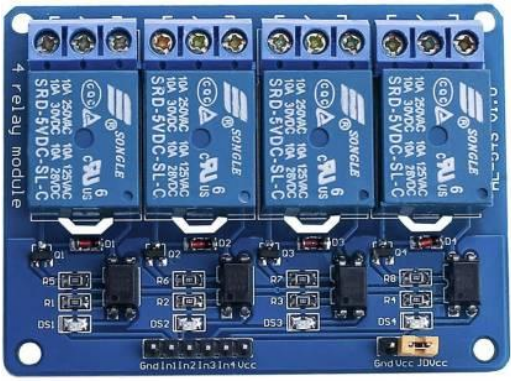

Figure 3: 4 Channel Relay

\section{Battery}

A battery is a device that consists of at least one electrochemical cell with external connections for controlling electrical devices such as lights, cell phones, and electric cars. The positive terminal of a battery is the cathode, and the negative terminal is the anode when it provides electric control. $12 \mathrm{~V} / 7.5$ $\mathrm{AH}$ battery is used in this project. Internal circuitry in these batteries allows them to be utilised as a drop-in replacement for sealed lead acid batteries. Lead acid chargers can be used to charge them. Because they do not require maintenance during the off-season, these batteries are ideal for use in motorcycles, snowmobiles, jet skis, and other recreational vehicles. These batteries can be charged manually using a power supply with voltage and current limits that may be adjusted by the user. The $12 \mathrm{v} 7.5 \mathrm{Ah}$ lead acid battery is a rechargeable power source. These batteries are made to deliver a large burst of electricity followed by a speedy recharging. This battery has six cells connected in series. Another benefit is that totally draining the battery does not harm it in any way.

\section{IR Temperature Sensor}

Thermopile sensors detect an object's infrared (IR) energy and use it to determine temperature from a distance. The more IR energy is emitted, the greater the temperature. Non-contact temperature is measured by Thermopile infrared (IR) temperature sensors. An infrared sensor does not emit a beam of light; instead, it uses infrared energy to measure the temperature that the target emits in a conical shape. MLX90614 infrared sensor is used in this project. The MLX90614 is an infrared thermometer that can be used to take non-contact temperature readings. The signal conditioning ASIC and the IR sensitive thermopile detector chip are both housed in the same TO-39 container. A low noise amplifier, 17-bit ADC, and powerful DSP unit are all built into the MLX90614 thermometer, resulting in great accuracy and resolution. The digital output can be set to pulse width modulation by the user (PWM). The 10-bit PWM is set up to continually communicate the measured temperature in the range of -20 to $120^{\circ} \mathrm{C}$, with an output resolution of $0.14^{\circ} \mathrm{C}$, as standard. The infrared sensor MLX90614 works by converting infrared radiation signals collected from objects and bodies into electrical signals, sending the electrical signals to a converter after noise amplification by an amplifier, then converting the electrical signal to digital signals and storing the processed signals in the internal memory, ultimately, transmit the signals to the SCM control system to be processed further.

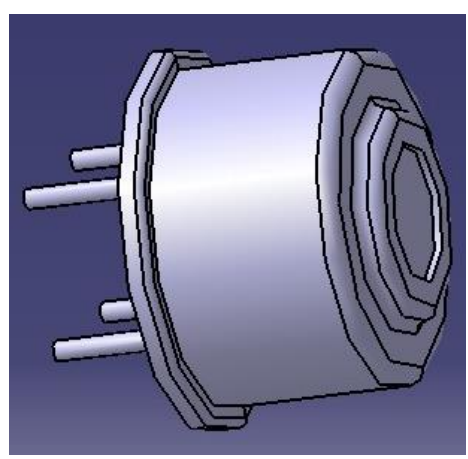

Figure 4: MLX90614 IR Sensor

\section{Sanitizer Pump}

A pump is a mechanical device that propels fluids (liquids or gases), or sometimes slurries, by converting electrical energy into hydraulic energy. Pumps are divided into three categories based on the technique of fluid movement which are directly lifting pumps, displacement pumps and gravitational pumps. Pumps 
use energy to produce mechanical work (usually reciprocating or rotational), and they consume it to move the fluid. Pumps can be propelled by manual labor, electricity, engines, or wind power, and they come in a wide range of sizes, from minuscule pumps for medical applications to large industrial pumps. A $12 \mathrm{~V} 60 \mathrm{~W}$ high pressure water pump is used in this project. Pumps are utilised for a variety of applications in modern civilization. The use of a windmill or a watermill to pump water was one of the first applications. Pumps are still used today for irrigation, water supply, petroleum supply, air conditioning systems, refrigeration (typically referred to as a compressor), chemical movement, sewage movement, flood control, and marine services, among other things. Pumps come in a wide range of shapes and sizes, from very large to extremely small, from handling gas to handling liquid, from high pressure to low pressure, and from high volume to low volume, due to the wide range of applications.

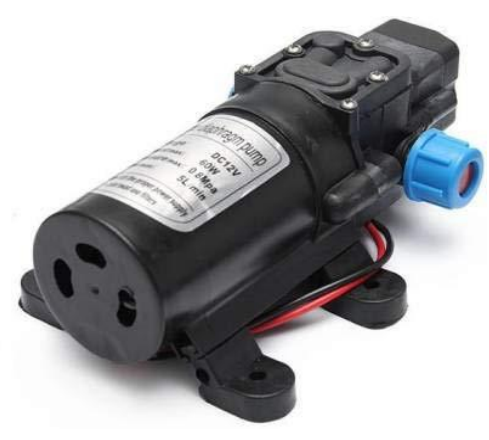

Figure 5: 12V Sanitizer Pump

\section{Framework}

This project's main framework is composed of steel with dimensions of $3 \times 2 \mathrm{ft}$. Steel is a naturally ductile material that responds to overload in a controlled and manageable manner. Steel frames can be customised in size and shape to give structural robustness that is difficult to achieve with other, more brittle construction materials. Steel offers the strength and support required for framework while maintaining the frame's desired weight. The framework gives the medicine tray and trash collecting chamber, as well as the Wi-Fi camera and sensors, the stability and strength they require. The framework is coated with paint to protect it from rust and corrosion.

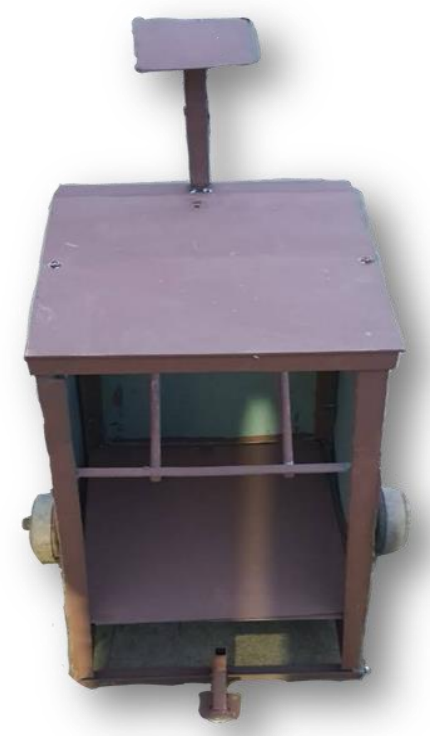

Figure 6: Steel Framework

\section{Wi-Fi Camera}

This camera is simple to use and can be put anywhere in the home (within wireless range of the internet connection) and operated remotely from smartphone or tablet. It records video in HD 720P at 30 frames per second and produces crystal clear images. It features a dual motor head that lets it to rotate and capture a 360-degree horizontal view as well as a 90-degree vertical view, allowing you to see your items from any angle. PAN \& TILT (LEFT, RIGHT, 355 DEGREES; UP, DOWN), a built-in microphone and speaker, a memory card adapter for recording video 24 hours a day on a memory card, and a plethora of other wonderful features. this is a basic monitoring tool for your own house or just to look at young people, seniors, or animals anywhere on the planet, this is the right option for you. Night vision provides a clear picture even in the dark. The powerful infrared night vision can see for a great distance and 
is easily noticeable at night. During the day and night, it makes clear, high-quality pictures. Simply set up and operate, just connect it to your smartphone / tablet and always have a live picture of anything.

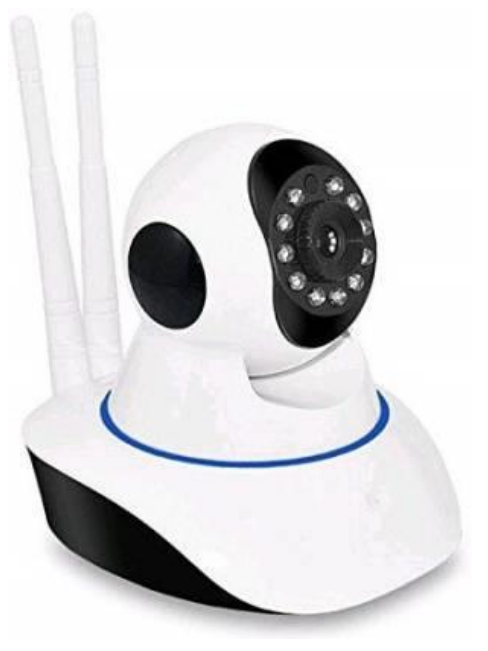

Figure 7: V380s WiFi Smart Camera

\section{GEOMETRIC MODELLING}

The components that will be utilized in these projects are 3D designed and drafted using the Catia V5 application based on the measurements. This provides us an idea of how the model will look when we've assembled all the components using the dimensions we've chosen.

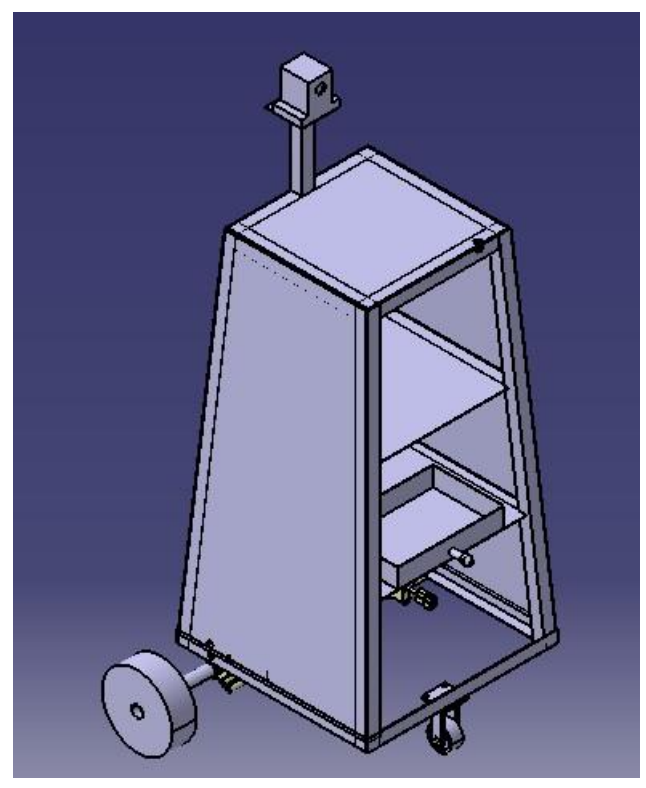

Figure 8: Prototype Assembly

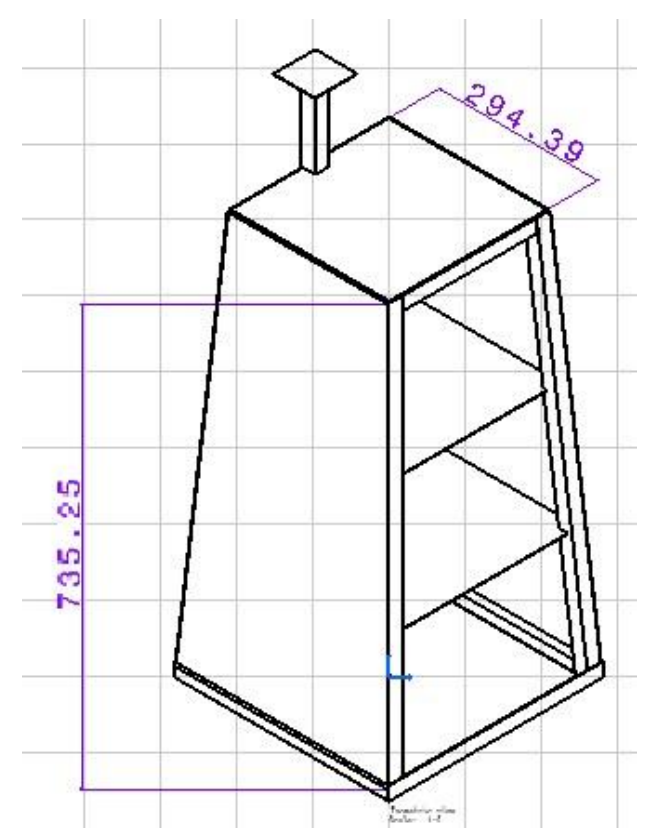

Figure 9: Framework Draft

\section{WORKING}

Entire vehicle is powered with $12 \mathrm{~V} 7.5 \mathrm{AH}$ battery, and a 60 -rpm drive motor to drive it, vehicle is controlled wirelessly with Wi-Fi module which gives max 50 metres range. Vehicle has 2 platforms; top platforms can carry medicine and food and bottom 
platform has waste collecting bin. Vehicle is controlled wirelessly using wireless visual from wireless camera, thus performing delivery of medicine, food and collecting waste and has IR Temperature sensor which provides Temperature of Patient to Doctor.

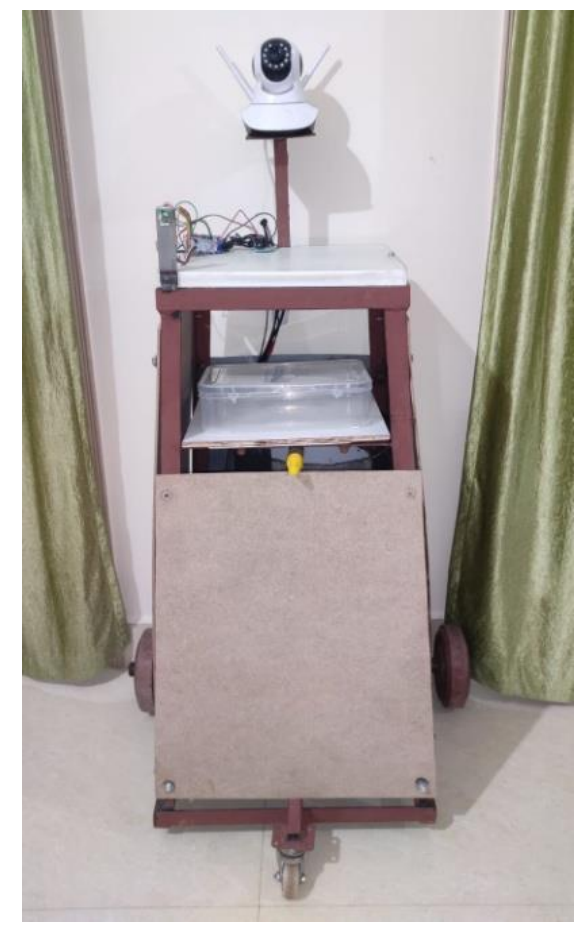

Figure 10: Prototype Final Assembly

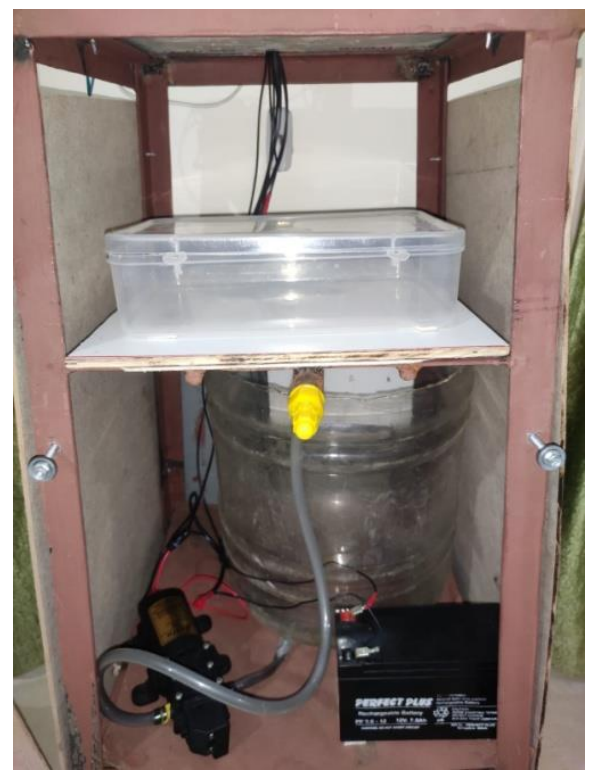

Figure 11: Sanitizer Dispenser and Medicine Tray Set
You can use Blynk to develop smartphone apps that allow you to interface with microcontrollers or even complete computers like the Raspberry Pi. The Blynk platform's primary goal is to make developing mobile phone applications as simple as possible. Creating a mobile app that can communicate with your Arduino is as simple as dragging a widget and setting a pin. With Blynk, you can control an LED or a motor from your phone with no programming required. Blynk innovation is the smartphone app. It gives you the option of creating one or more projects. Each project may include graphical widgets such as virtual LEDs, buttons, value displays, and even a text terminal, as well as interface with one or more devices. It is possible to control Arduino or ESP32 pins straight from your phone using the Blynk library, without having to write any code at all. It is also possible to share a project with friends or even clients, allowing them to access the linked devices but not alter the project.

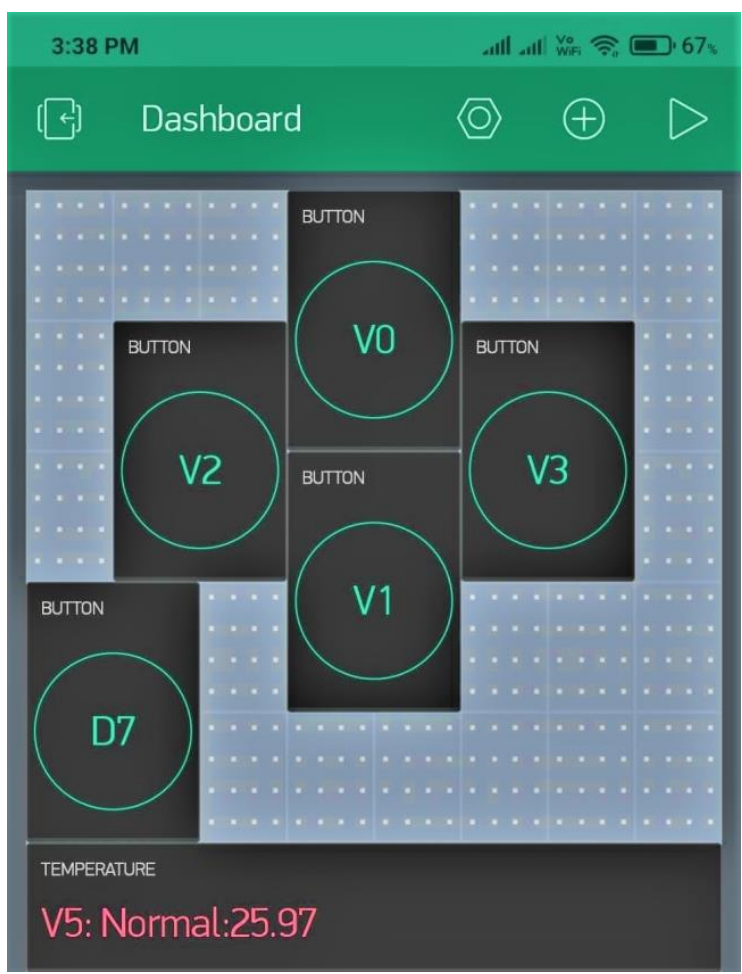

Figure 12: BLYNK App User Interface

\section{BLYNK}

\section{V380s Wi-Fi Camera Application}


The V380s is a new generation of intelligent household cloud camera free application that allows for easy remote video monitoring and administration.

1. This programme allows you to see the real-time video process at any time and from any location.

2. Remote PTZ control is supported, with camera rotation accomplished by tapping the screen.

3. Allow for network-wide live audio monitoring.

4. Network remote video playback and picture capture are supported.

5. Enable site motion detection alarms and save the server to see.

6. Voice intercom and video calls are supported.

7. Intelligent cloud streaming transmission technology with million high-definition public network real-time transport.

8. Improved digital zoom, preset, and WIFI Smart link setting functions. Support for fast AP configuration; two-dimensional code scanning device ID, and other features.

9. Live Preview improves the recording feature, allowing you to see the recorded video in an album.

10. Video files improve the download operation, allowing you to study the downloaded video in an

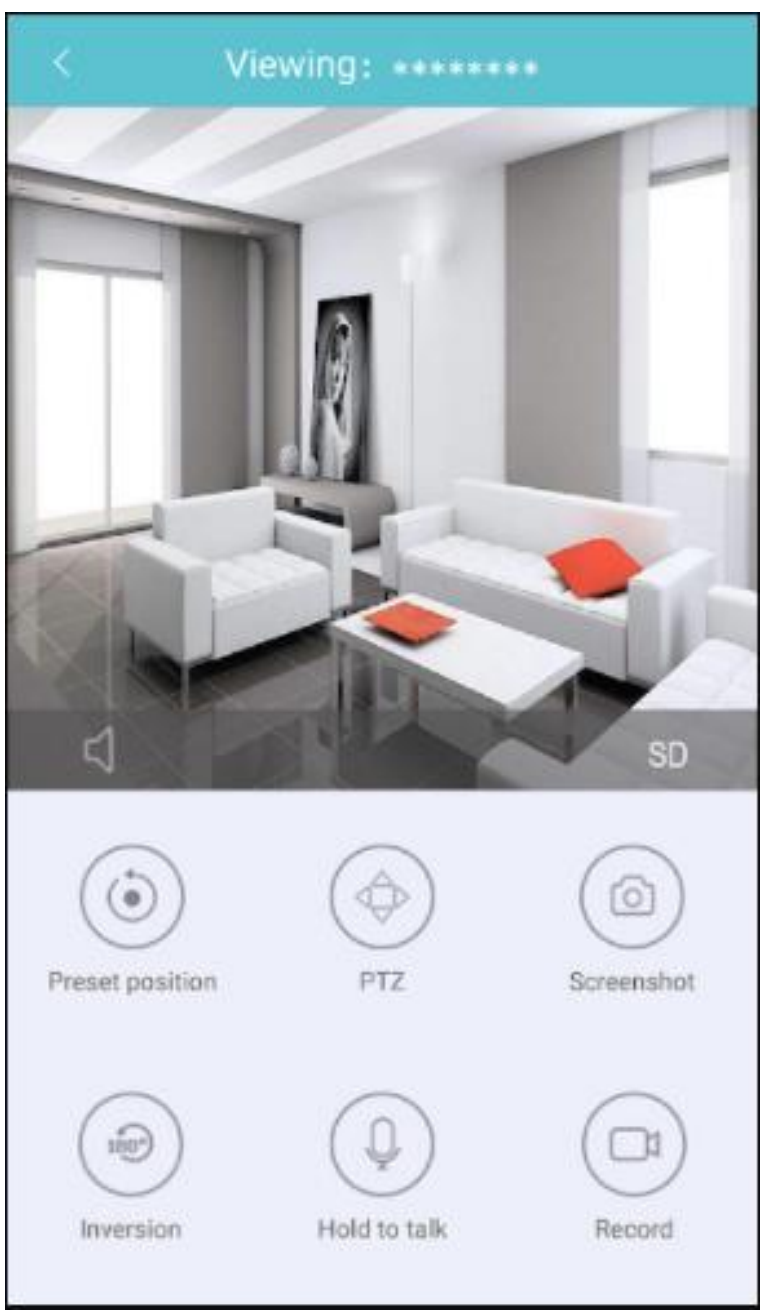

Figure 13: V380s App User Interface album.

11. Expands cloud storage services, cloud services link devices, video may be uploaded to the server, and data security is improved.

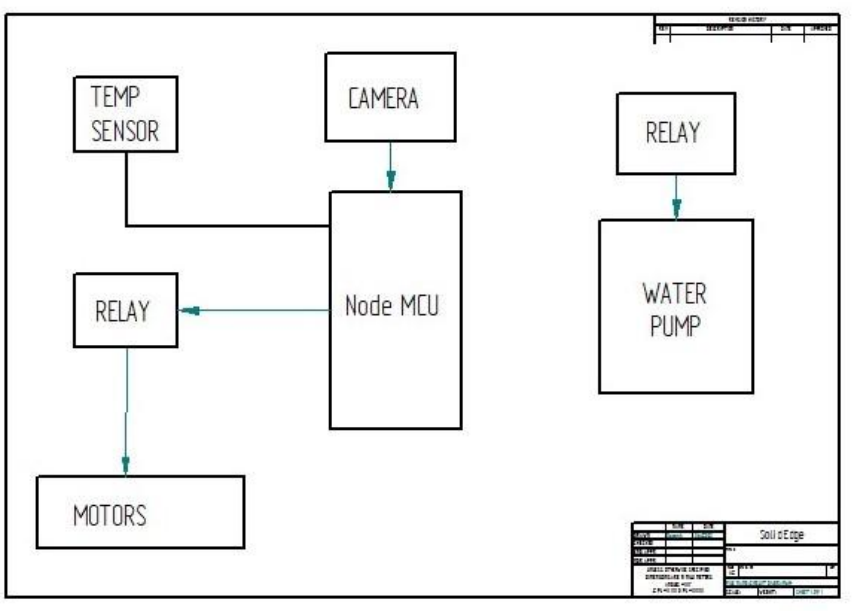

Figure 14: Working Block Diagram

V. BILL OF MATERIALS

Bill of Material 


\begin{tabular}{|c|l|c|c|}
\hline Sl. No. & \multicolumn{1}{|c|}{ Title } & Quantity & Cost \\
\hline 1 & Main frame & 1 NOs & 2500 \\
\hline 2 & Drive motor & 2 NOs & 5000 \\
\hline 3 & Wheels & 3 NOs & 1200 \\
\hline 4 & Controller & 1 NOs & 500 \\
\hline 5 & Camera & 1 NOs & 1300 \\
\hline 6 & Battery & 1 NOs & 900 \\
\hline 7 & $\begin{array}{l}\text { Vehicle body } \\
\text { cover }\end{array}$ & 7 NOs & 600 \\
\hline 8 & IR temp sensor & 1 NOs & 1000 \\
\hline 9 & Relay & 5 NOs & 200 \\
\hline & \multicolumn{2}{|c|}{ Total } & 13,200 \\
\hline
\end{tabular}

\section{RESULTS AND DISCUSSION}

\section{Testing of Project}

Various sorts of tests and experiments are intended to validate the project's capacity to sustain real-world activity, and the findings are recorded to assert that the project's objectives are met.

\section{A) Working Limits of Project}

The working hour limit of the project was tested, and it was observed that, the project could run/ operated continuously without any interruptions for duration of 90-120 minutes when the battery (12V 7.5ah) was fully charged. Time required for fully charging the battery was found to be approximately 60-90 minutes.

The effective/safe ESP8266 Wi-Fi range for controlling the project's movements was tested and found to be: frequency range: 2400-2484 MHz; distance range: 50-100 metres.

\section{B) Speed Test}

The speed test in rotation per minute (RPM) was conducted on the project and the results are tabulated below:

Motor specification:

Operating voltage $=10-12$ V DC; Current supply $=20$ Amps.

Wheel dimensions:

Radius $=0.07 \mathrm{~m}$.

Circumference $=2^{*} \mathrm{Pi}^{*}$ radius $=2 * 3.141^{*} 0.07=0.44 \mathrm{~m}$.

$\begin{array}{cc}\text { Test Cycles } & \text { Rotation Per Minute (RPM) } \\ \text { Test Run 1 } & 23 \\ \text { Test Run 2 } & 25 \\ \text { Test Run 3 } & 24 \\ \text { Test Run } 4 & 28 \\ \text { Test Run 5 } & 26\end{array}$

Hence, the average speed in RPM was found to be 25 Rpm.

\section{C) Load Test}

Total Estimated weight of the project: $15 \mathrm{Kgs}$ After applying a series of load on the project, it was concluded that the Safe load carrying capacity of the project for seamless operation must be below $7 \mathrm{Kgs}$.

\section{D) Sensor Accuracy Test}

Test was conducted to check the accuracy and precision of the Sensor based on temperature measurement. The comparison between the readings of the IR sensor and a medical thermometer were done and the results are tabulated below: 
Sensor used for temperature measurement: Calculation:

MLX90614 infrared sensor

Specifications:

Temperature measurement range $=-20$ to $120^{\circ} \mathrm{C}$.

Output resolution $=0.14^{\circ} \mathrm{C}$.

Operating voltage $=3-5 \mathrm{~V}$.

Accuracy $\%=(($ Thermometer reading - IR Sensor Reading) *100)/ Thermometer reading.

TABLE II

Sensor Accuracy Test Readings

\begin{tabular}{|c|c|c|c|}
\hline $\begin{array}{c}\text { Thermometer } \\
\text { Reading } \\
\left({ }^{\circ} \mathrm{c}\right)\end{array}$ & $\begin{array}{c}\text { IR Sensor } \\
\text { Reading } \\
\left({ }^{\circ} \mathrm{c}\right)\end{array}$ & Remarks & $\begin{array}{c}\text { Accuracy } \\
(\%)\end{array}$ \\
\hline 35 & 34.7 & Normal & 99.2 \\
\hline 39 & 39 & Fever & 100 \\
\hline 38.6 & 38.5 & Fever & 99.8 \\
\hline 36 & 36.2 & Normal & 99.5 \\
\hline
\end{tabular}

Hence, the accuracy and precision of the IR Sensor was checked and found out to be satisfactory according to the objective of the project with real time indication of the patient's temperature level whether he/she is 'NORMAL' or has a 'FEVER'.

\section{E) Sanitization Range Test}

Here the pump used for sanitization has the following specifications:

EARTH 12 V 60 W Pump.

Operating voltage $=12 \mathrm{~V}$.

Current supply $=3$ Amps.

Flow rate $=5$ litres per minute.

Pressure $=110$ PSI (6.8 Bar)

Sanitizer Tank capacity $=10$ litres.

Minimum liquid level to achieve required pressure $=$

2-3 inches above the outlet pipe of tank.

Nozzle: Pesticide Nozzle with adjustable head to control the discharge. It has three modes of spraying (LOW/ MEDIUM /HIGH).
Assuming, the area of sanitization is a circle with a diameter of $0.6 \mathrm{~m}$.

With MEDIUM spray configuration, the area sanitized in 3 seconds is equal to

$\left(\mathrm{Pi}^{*}\left(\mathrm{dia}^{2}\right)\right) / 4=\left(3.141^{*}\left(0.6^{2}\right)\right) / 4=\mathbf{0 . 2 8 3} \mathrm{m}^{2}$.

Test Floor space area $=\mathbf{1 5 . 1 2} \mathrm{m}^{2}$.

Hence, it takes 160 seconds (i.e., 2minutes 40 seconds) to sanitize the test floor area completely.

\section{F) Wi-Fi Camera Quality Test}

The Wi-Fi camera used in this project has the following specification:

V380s Smart WiFi Camera

HD Video Resolution format $=1.3$ megapixels.

Video Resolution $=1280 \times 1024$; Aspect ratio = 5:4.

Recording frame rate $=30$ FPS

Angle of View $=360$ degrees $(\mathrm{Pan}$ and Tilt $)$

The camera was tested, and the resolution of the video was found to be clear and distinct with Audible playback speakers.

\section{CONCLUSION}

COVID-19, a global pandemic disease, reminded us about the importance of being prepared for a pandemic disease outbreak in the near future. In these times of pandemic, frontline healthcare workers are at high risk and susceptible to infection. To protect, aid and support them, we therefore intend to construct a nursing vehicle which can be used in hospital surroundings. The number of infected patients and casualties can be considerably reduced if COVID-19 is effectively managed. The goal of this project is to maintain proper patient monitoring, delivery of needed supplies, and sanitization of the hospital environment even when humans are not present. Automation is a technique that is now widely 
used in practically all sectors, ranging from intricate rocket technology to crop monitoring in the realm of agriculture. There are numerous applications for automation in the healthcare industry.

Contactless temperature measurement and supply delivery to contagious disease patients will be possible with the nursing vehicle. This project consists of WiFi camera, water pump, temperature sensor, microcontroller, and embedded $\mathrm{C}$ language program. The nursing vehicle not only explains the fundamental ideas of automation, but it also connects hardware and software, demonstrating how this may be easily managed using microcontrollers and other components. The project is locally controlled by the use of ESP8266 based embedded system. The main frame is made up of steel and $12 \mathrm{~V} 60 \mathrm{RPM}$ drive motor is used for driving. The MLX90614 IR temperature sensor is also included, which aids in the measurement of patient temperature. The project also has nozzle and pump kit for sanitizing process. The motions of the nursing vehicle are controlled by a Wi-Fi controller and monitored by a camera that also contains a speech kit for interactions between patients and doctors. The project is selected thinking about the drawbacks faced in hospital environments. In these times of pandemic, the nursing vehicle will be extremely important in ensuring the safety of healthcare workers and addressing issues such as nurse and doctor shortages. It can be concluded that with correct connection of some simple hardware and software components, it is possible to create an automated vehicle which can be very useful in hospitals in this pandemic by reducing burden on healthcare staffs and increasing chances of their safety.

\section{REFERENCES}

[1]. Johan Potgieter, Glen Bright, Olaf Diegel, Sylvester Tlale, "Internet Control of a Domestic Robot Using a Wireless Lan" ARAA 2002, Auckland.
[2]. F. Capezio F. Mastrogiovanni, A. Scalmato, A. Sgorbissa, P. Vernazza, T. Vernazza, R. Zaccaria, "Mobile Robots in Hospital Environments: an Installation Case Study",ECMR 2011.

[3]. David Feil-Seife, Maja J Mataric, "Defining Socially Assistive Robotics”,IEEE 2005, USA.

[4]. Ali Gurcan Ozkil, Ali Gurcan; Fan, Zhun; Dawids, Steen; Klaestrup Kristensen, Jens; Christensen, Kim Hardam; Aanaes, Henrik, "Service Robots for Hospitals: A Case Study of Transportation Tasks in a Hospital”, IEEE 2009, China.

[5]. Seohyun Jeon, Jaeyeon Lee, "Multi-Robot MultiTask Allocation for Hospital Logistics", ICACT 2016, Korea.

[6]. Azeta Joseph, Bolu Christian, Abioye A. Abiodun, Festus Oyawale, "A review on humanoid robotics in healthcare", MATEC Web of Conferences 153, 02004 (2018), ICMME 2017.

[7]. Dr.K.Lakshmi Narayanan, Dr.N.Muthukumaran, Dr.G.Rajakumar, Mr. Hameedhul Arshadh, Mr.I.Dinesh, Mr. V.Caleb, "Design and Fabrication of Medicine Delivery Robots for Hospitals", ICRTCCNT 2019,India.

[8]. Simon Thiel, Dagmar Häbe, Micha Block,"Cooperative Robot Teams in a Hospital Environment", IEEE 2009,Germany.

\section{Cite this article as :}

Gopal Kaliyaperumal, Choudhury Rajat Kumar Pattnaik, Akash Kumar Nath, Aditya Dubey, Tarun Kumar Sharma, "Design and Fabrication of RemoteControlled Nursing Vehicle", International Journal of Scientific Research in Science, Engineering and Technology (IJSRSET), Online ISSN : 2394-4099, Print ISSN : 2395-1990, Volume 8 Issue 4, pp. 188-198, July-August 2021. Available at doi : https://doi.org/10.32628/IJSRSET218434 Journal URL : https://ijsrset.com/IJSRSET218434 